\title{
Left ventricular volumes and function affected by myocardial fibrosis in patients with Duchenne and Becker muscular dystrophies: a preliminary magnetic resonance study
}

\author{
Joanna Kwiatkowska', Jarosław Meyer-Szary', Magdalena Bazgier'1, Jadwiga Fijałkowska², \\ Jolanta Wierzba ${ }^{3}$, Anna Glińska², Karolina Dorniak ${ }^{4}$ \\ 1 Department of Paediatric Cardiology and Congenital Heart Defects, Medical University of Gdańsk, Gdańsk, Poland \\ 2 2nd Department of Radiology, Medical University of Gdańsk, Gdańsk, Poland \\ 3 Department of Paediatrics, Haematology and Oncology, Department of General Nursery, Medical University of Gdańsk, Gdańsk, Poland \\ 4 Department of Noninvasive Cardiac Diagnostics, Medical University of Gdańsk, Gdańsk, Poland
}

Correspondence to: Karolina Dorniak, MD, PhD, Department of Noninvasive Cardiac Diagnostics, Medical University of Gdańsk, Poland, ul. Dębinki 7, 80-211 Gdańsk, Poland phone: +48583493380 , email: karolina.dorniak@gumed.edu.pl Received: November 11, 2019

Revision accepted: February 24, 2020.

Published online: March 2, 2020. Kardiol Pol. 2020; 78 (4): 331-334 doi:10.33963/KP.15223 Copyright by the Author(s), 2020
Introduction Cardiac magnetic resonance (CMR) provides means for tissue characterization and has been used in different populations to assess global and regional functions, presence of edema, inflammation, necrosis, and fibrosis of the heart chambers. ${ }^{1-4}$ Early cardiac involvement can be shown in CMR when standard cardiovascular workup including 12-lead electrocardiography, 24-hour electrocardiography, and echocardiography are normal-appearing. ${ }^{5}$ The early identification of cardiac involvement in patients with Duchenne muscular dystrophy (DMD) is important as it allows for a timely introduction of cardioprotective therapy to slow down the progression of heart failure and to reduce symptoms. ${ }^{6}$ Based on scarce data from limited cohort studies, it is the fibrosis that is the most frequent and relevant abnormality evolving throughout patients' lifetime.

The aim of the study was to characterize cardiac involvement in patients of all age groups with DMD and Becker muscular dystrophy (BMD).

Methods We present a single center cross-sectional observational study of the patients representing the DMD population from the entire country.

Inclusion and exclusion criteria as well as detailed CMR diagnostic procedure and protocol are presented in Supplementary material.
The study was approved by the institutional bioethical committee and all patients' guardians gave their informed consent.

Statistical analysis was performed using the Wizard Pro version 1.9.33 (Evan Miller, Chicago, Illinois, United States). Continuous variables are presented as mean (SD) or median (range) depending on the distribution. The $\mathrm{X}^{2}$ test, Pearson correlations, and Spearman rank correlations were employed.

Results and discussion Out of 79 screened patients, 41 were enrolled after applying inclusion and exclusion criteria and were successfully examined using CMR. They were all men at a mean (SD) age of 12 (3.1) years. A total of 37 patients (90.2\%) had DMD, and 4 (9.8\%) had $\mathrm{BMD}$. The mean (SD) left ventricular (LV) end-diastolic volume index was $63.6(17.4) \mathrm{ml} / \mathrm{m}^{2}$ and was decreased in $24 \%$ of patients. ${ }^{7}$ The mean (SD) LV end-systolic volume index (LVESVI) was 30 (9) $\mathrm{ml} / \mathrm{m}^{2}$ and was abnormally high in $12 \%$ and abnormally low in $2 \%$ of patients. The mean (SD) LV stroke volume index (LVSVI) was $37(10.8) \mathrm{ml} / \mathrm{m}^{2}$ and was abnormally low in $39 \%$ of patients. The mean (SD) LV ejection fraction (LVEF) was $58 \%$ (6.4\%) and was low in $44 \%$ of patients. Older patients had significantly lower LV end-diastolic volume index $z$ score $(r=-0.41, P=0.008$; FIGURE 1A) but not LVESVI 

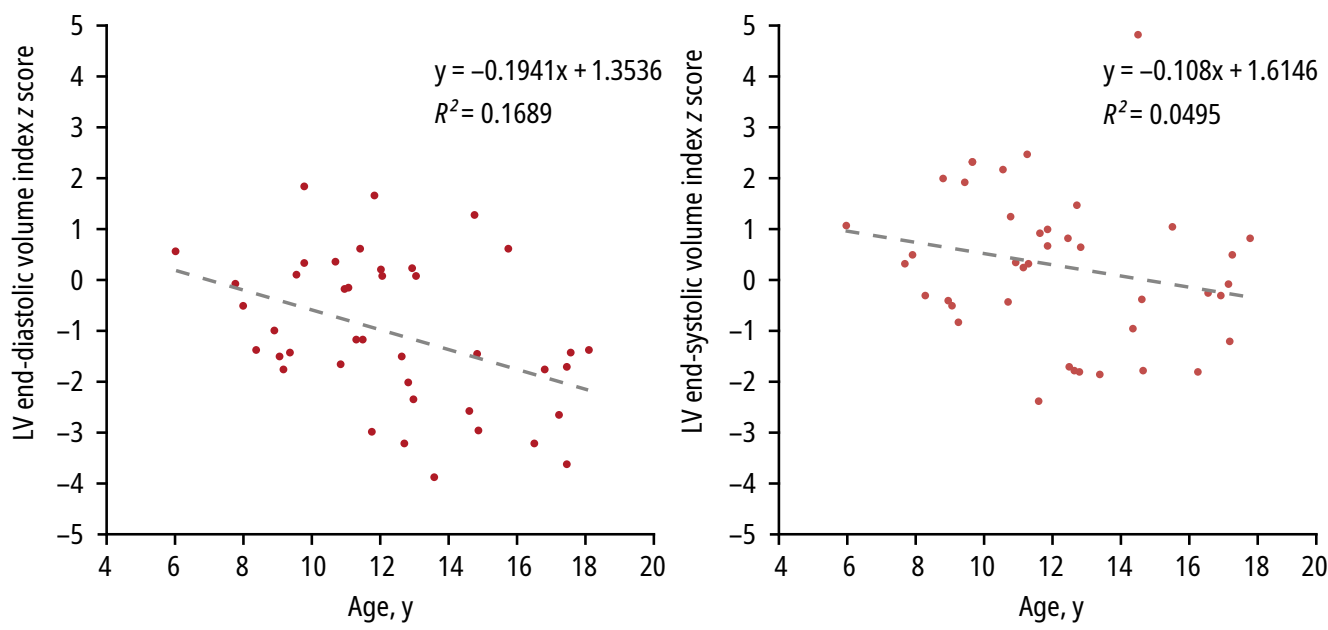

c

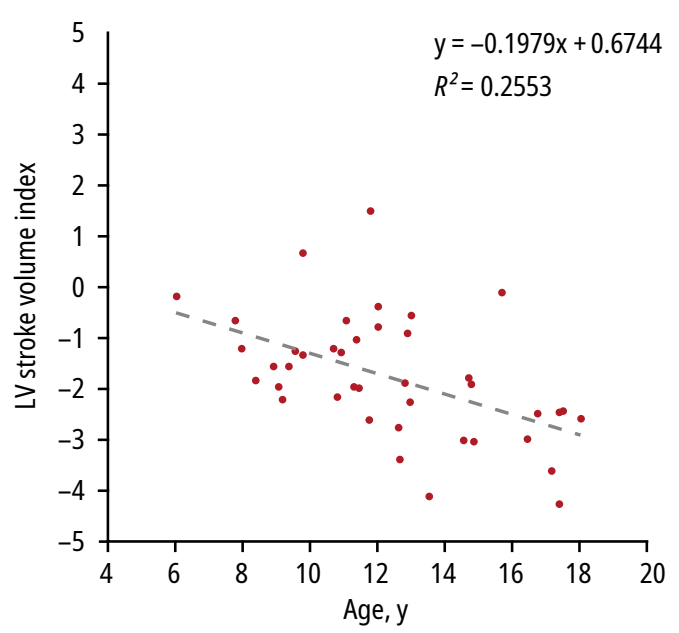

西

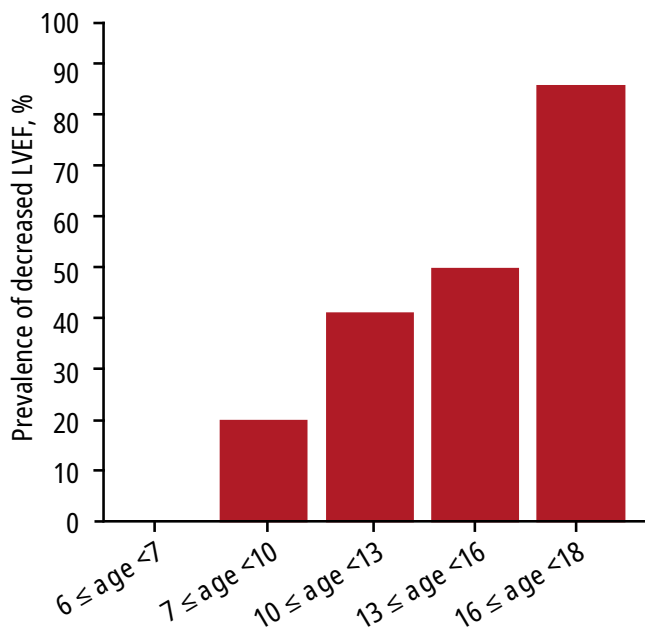

Age group
D

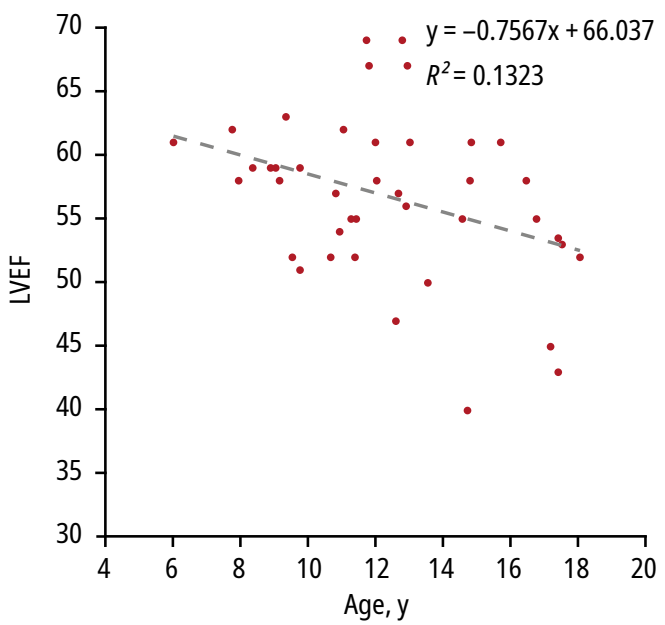

$\mathbf{F}$

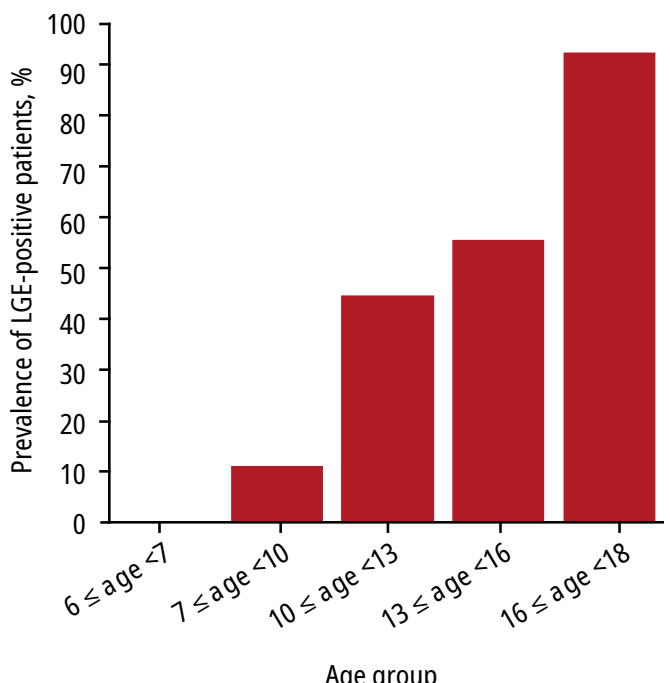

Age group

FIGURE 1 The scatter plots show the correlations of left ventricular (LV) volume-related parameters with age: end-diastolic volume (A), end-systolic volume (B), stroke volume (C), and LV ejection fraction (LVEF; D). The bar charts represent the prevalence of abnormalities found in different age groups: decreased $(<55 \%)$ left ventricle ejection fraction $(\mathbf{E})$, and patients with at least 1 segment positive for late gadolinium enhancement (LGE) (F). 
$z$ score $(P=0.16$; FIGURE 1B). Consequently, also negatively correlated with age were LVSVI $z$ score $(r=-0.50, P<0.001$; FIGURE 1 c $)$ and LVEF $(r=-0.36$, $P=0.02$; FIGURE 1D). Also, the prevalence of patients with decreased LVSVI (Supplementary material, Figure S1A), and decreased LVEF (FIGURE 1E) was higher in older age groups $(P=0.001$ and $P=0.04$, respectively).

Late gadolinium enhancement (LGE) was assessed in 39 patients and was positive in $38 \%$ of them, most often in the midanterolateral (38\%), basal-anterolateral (36\%), basal-inferolateral (31\%), mid-inferolateral (26\%), and apical-lateral (18\%) segments (Supplementary material, Figure S2). In 2 patients, LGE images were deemed nondiagnostic due to massive respiratory artifacts. LGE was significantly more prevalent in the older age groups ( $P=0.02$; FIGURE 1F). Also, the fibrosis extensiveness correlated positively with age ( $r=0.036, P=0.02$; Supplementary material, Figure $S 1 B)$. Noticeably, no LGE was found in any of patients with BMD. The patients with positive LGE had significantly lower LVSVI $z$ score (mean [SD], -2.3 [1.0] vs -1.3 [1.1]; $P=0.02$ ) and LVEF (53.2 [5.5] vs 59.5 [5.3]; $P<0.001$ ). Furthermore, the extent of fibrosis, irrespective of its pattern, correlated with decreased LVEF $(P<0.001, r=-0.531$; Supplementary material, Figure S1C). More detailed data can be found in Supplementary material (Tables S1-S4).

Our study presents preliminary cross-sectional data from a Polish cohort of patients with DMD and BMD. In line with the previously published data, ${ }^{8,9}$ in patients with $\mathrm{DMD}$, we were able to show that LGE of the LV myocardium is associated with reduced LVEF. Moreover, our study confirmed that the extent of cardiac involvement increases with the disease progression resulting from advancing age, as previously demonstrated. ${ }^{10}$ It is known that in DMD and BMD, myocardial necrosis starts from the posterobasal region of the left ventricle, progressing to other cardiac segments and leading to heart remodeling, which is partly in line with our findings, where posterobasal and basal anterolateral regions of the LV were commonly involved, including the patients with LGE of lesser extent.

Contrary to the previously published studies that suggested LV dilatation coupled with the reduction of LVEF, ${ }^{10}$ our findings showed decreased end-diastolic volumes with preserved end-systolic volumes, resulting in decreased stroke volume and LVEF, a pattern of involvement distinct from other cardiomyopathies. Enlargement of the LV was not noted as the study described young population in an early stage of the disease whereas LV dilatation and overt heart failure tends to develop in the third decade of life in the majority of cases.

The presence, extent, and distribution of LGE and its relation to LV function as assessed by
LVEF in patients with muscular dystrophy were previously studied by a number of authors. ${ }^{11,12}$ In a study by Brunklaus et al, ${ }^{12}$ extensive but not minimal LGE was associated with reduced LVEF ( $48 \%$ vs $58 \%$, respectively), suggesting more severe cardiomyopathy. ${ }^{12}$ Our study confirms the correlation between the presence of any fibrosis and decrease in LVEF. However, the cardiac function may be preserved for many years in DMD, even with fibrosis progression..$^{11,13}$ Moreover, whether LGE extent at baseline predicts the speed of cardiac function impairment over the following years warrants further investigation. Given the limitations of physical activity with age in DMD, the affected individuals may not display clinical symptoms unless they are exposed to additional stress. ${ }^{14}$

Finally, all the dot plots also show a noticeable variability among the patients, (also of similar age), suggesting an uneven cardiac involvement in patients. This observation provides the basis for further longitudinal studies in the search for CMR parameters allowing for risk stratification and treatment escalation for those at risk.

In conclusion, in patients with muscular dystrophy, fibrosis advances with age and is related with impaired LV function. Cardiac magnetic resonance provides a detailed insight in chamber volumes, myocardial function, and tissue characterization, all of which allow for the detection of subtle subclinical cardiac involvement. Therefore, it may become a useful aid in determining the early cardioprotective therapy.

\section{SUPPLEMENTARY MATERIAL}

Supplementary material is available at www.mp.pl/kardiologiapolska.

\section{ARTICLE INFORMATION}

\section{CONFLICT OF INTEREST None declared.}

OPEN ACCESS This is an Open Access article distributed under the terms of the Creative Commons Attribution-NonCommercial-NoDerivatives 4.0 International License (CC BY-NC-ND 4.0), allowing third parties to download articles and share them with others, provided the original work is properly cited, not changed in any way, distributed under the same license, and used for noncommercial purposes only. For commercial use, please contact the journal office at kardiologiapolska@ptkardio.pl.

HOW TO CITE Kwiatkowska J, Meyer-Szary J, Bazgier M, et al. Left ventricular volumes and function affected by myocardial fibrosis in patients with Duchenne and Becker muscular dystrophies: a preliminary magnetic resonance study. Kardiol Pol. 2020; 78: 331-334. doi:10.33963/KP.15223

\section{REFERENCES}

1 Miłosz-Wieczorek B, Kłopotowski M, Kubik A, et al. Quantitative oedema analysis using cardiac magnetic resonance T2-mapping techniques in diagnosis of takotsubo cardiomyopathy. Kardiol Pol. 2017; 75: 1356.

2 Haberka M, Machnik G, Kowalówka A, et al. Epicardial, paracardial, and perivascular fat quantity, genes expressions, and serum cytokines in patients with coronary artery disease and diabetes. Pol Arch Intern Med. 2019; 129: 738-746.

3 Glaveckaite S, Palionis D, Valeviciene N, et al. Intramyocardial haemorrhage as a rare complication of myocardial infarction - the diagnostic value of cardiovascular magnetic resonance imaging. Kardiol Pol. 2017; 75: 934.

4 Ziółkowska L, Śpiewak M, Małek $Ł$, et al. The usefulness of cardiovascular magnetic resonance imaging in children with myocardial diseases. Kardiol Pol. 2015; 73: 419-428.

5 Petkow-Dimitrow P, Tomkiewicz-Pająk L, Karpiński M, et al. Cardiac magnetic resonance imaging in a woman suspected of hypertrophic cardiomyopathy based on genotyping. Pol Arch Intern Med. 2018; 128: 617-618. 
6 Lamacie MM, Warman-Chardon J, Crean AM, et al. The Added Value of Cardiac Magnetic Resonance in Muscular Dystrophies. J Neuromuscul Dis. 2019; 6: 389-399.

7 Kawel-Boehm N, Maceira A, Valsangiacomo-Buechel ER, et al. Normal values for cardiovascular magnetic resonance in adults and children. J Cardiovasc Magn Reson. 2015; 17: 29 .

8 Hor KN, Taylor MD, Al-Khalidi HR, et al. Prevalence and distribution of late gadolinium enhancement in a large population of patients with Duchenne muscular dystrophy: effect of age and left ventricular systolic function. J Cardiovasc Magn Reson. 2013; 15: 107

9 D'Amario D, Amodeo A, Adorisio R, et al. A current approach to heart failure in Duchenne muscular dystrophy. Heart. 2017; 103: 1770-1779.

10 Melacini P, Vianello A, Villanova C, et al. Cardiac and respiratory involvement in advanced stage Duchenne muscular dystrophy. Neuromuscul Disord. 1996; 6: 367-376.

11 Tandon A, Villa CR, Hor KN, et al. Myocardial Fibrosis Burden Predicts Left Ventricular Ejection Fraction and Is Associated With Age and Steroid Treatment Duration in Duchenne Muscular Dystrophy. J Am Heart Assoc. 2015; 4: 1-8.

12 Brunklaus A, Parish E, Muntoni F, et al. The value of cardiac MRI versus echocardiography in the pre-operative assessment of patients with Duchenne muscular dystrophy. Eur J Paediatr Neurol. 2015; 19: 395-401.

13 Puchalski MD, Williams RV, Askovich B, et al. Late gadolinium enhancement: precursor to cardiomyopathy in Duchenne muscular dystrophy? Int J Cardiovasc Imaging. 2009; 25: 57-63.

14 Doltra A, Stawowy P, Dietrich T, et al. Magnetic resonance imaging of cardiovascular fibrosis and inflammation: from clinical practice to animal studies and back. BioMed Res Int. 2013: 676489. 